\title{
ON BIRATIONAL MONOMIAL TRANSFORMATIONS OF PLANE
}

\author{
ANATOLY B. KORCHAGIN
}

Received 20 June 2003 and in revised form 7 October 2003

\begin{abstract}
We study birational monomial transformations of the form $\varphi(x: y: z)=\left(\varepsilon_{1} x^{\alpha_{1}} y^{\beta_{1}} z^{\gamma_{1}}\right.$ : $\left.\varepsilon_{2} x^{\alpha_{2}} y^{\beta_{2}} z^{\gamma_{2}}: x^{\alpha_{3}} y^{\beta_{3}} z^{\gamma_{3}}\right)$, where $\varepsilon_{1}, \varepsilon_{2} \in\{-1,1\}$. These transformations form a group. We describe this group in terms of generators and relations and, for every such transformation $\varphi$, we prove a formula, which represents the transformation $\varphi$ as a product of generators of the group. To prove this formula, we use birationally equivalent polynomials $A x+B y+C$ and $A x^{p}+B y^{q}+C x^{r} y^{s}$. If $\varphi$ is the transformation which carries one polynomial onto another, then the integral powers of generators in the product, which represents the transformation $\varphi$, can be calculated by the expansion of $p / q$ in the continued fraction.
\end{abstract}

2000 Mathematics Subject Classification: 14E07, 14L30.

1. Introduction. Birational monomial transformations of the projective plane have already found a lot of applications. For example, such transformations are actively used for construction of real algebraic curves and surfaces (see, e.g., [1, 4, 5, 6, 8, 9]). We think that formula (3.6) will be helpful for description of construction of algebraic objects.

In Section 2 we give a little exposition on projective polynomials in three variables. In Section 3 we describe the birational monomial group in terms of its generators and relations and give the statement of a theorem of decomposition of birational monomial transformations. In Section 4 we give the proof of the theorem.

2. Preliminaries. A nonzero homogeneous polynomial of degree $n$ in three variables, $x, y, z$, is the expression

$$
f(x, y, z)=\sum_{\omega_{1}+\omega_{2}+\omega_{3}=n} f_{\omega} x^{\omega_{1}} y^{\omega_{2}} z^{\omega_{3}}, \quad \omega=\left(\omega_{1}, \omega_{2}, \omega_{3}\right) .
$$

The convex hull of the set $\left\{\left(\omega_{1}, \omega_{2}\right) \in \mathbb{R}^{2} \mid f_{\omega} \neq 0\right\}$ is called the Newton polygon of the polynomial $f(x, y, z)$ and is denoted as $N(f)$. The plane with coordinates $\left(\omega_{1}, \omega_{2}\right)$ is called the plane of Newton's polygons.

Every polynomial $f(x, y, 1)$ can be represented in the form $f(x, y, 1)=x^{i} y^{j} f^{\wedge}(x, y$, 1 ), where $i$ and $j$ are nonnegative integers, and the polynomial $f^{\wedge}(x, y, 1)$ has no factors $x$ and $y$. If $\varphi$ is a transformation, then clearly $\left(f^{\wedge} \circ \varphi\right)^{\wedge}=(f \circ \varphi)^{\wedge}$. It is also clear that the Newton polygon $N\left(f^{\wedge}\right)$ can be obtained from the Newton polygon $N(f)$ by translation in the plane of Newton's polygons by the vector $(-i,-j)$.

3. The birational monomial group. Let $(x: y: z)$ be homogeneous point coordinates in the projective plane $\mathbb{K} P^{2}$ over a field $\mathbb{K}$ and let $(x, y)$ be affine coordinates in the affine chart $\mathbb{K}^{2}=\mathbb{K} P^{2} \backslash\{z=0\}$. A projective transformation $\varphi$ is defined by the 
formula $\varphi(x: y: z)=\left(\varphi_{1}(x, y, z): \varphi_{2}(x, y, z): \varphi_{3}(x, y, z)\right)$, where $\varphi_{1}, \varphi_{2}, \varphi_{3}$ are homogeneous polynomials of the same degree, assumed to have no common factors. For the transformation $\varphi(x: y: z)$, we define its natural restriction $\varphi(x, y)$ to the affine chart $\mathbb{K}^{2}=\mathbb{K} P^{2} \backslash\{z=0\}$ by the formula $\varphi(x, y)=\left(\varphi_{1}(x, y, 1) / \varphi_{3}(x, y, 1), \varphi_{2}(x, y\right.$, 1) $\left./ \varphi_{3}(x, y, 1)\right)$.

Let id : $\mathbb{K} P^{2} \rightarrow \mathbb{K} P^{2}$ be the identity map. If $\varphi$ is a birational transformation, then we denote as usual

$$
\varphi^{0}=\mathrm{id}, \quad \underbrace{\varphi \circ \cdots \circ \varphi}_{n \text { times }}=\varphi^{n}, \quad \underbrace{\varphi^{-1} \circ \cdots \circ \varphi^{-1}}_{n \text { times }}=\varphi^{-n} .
$$

Let $r_{1}, r_{2}, r_{3}: \mathbb{K} P^{2} \rightarrow \mathbb{K} P^{2}$ be maps defined by formulas $r_{1}(x: y: z)=((-x): y: z)$, $r_{2}(x: y: z)=(x:(-y): z)$, and $r_{3}(x: y: z)=(x: y:(-z))$. The set of maps $R=$ $\left\{\right.$ id, $\left.r_{1}, r_{2}, r_{1} \circ r_{2}\right\}$ with the operation of composition of the maps, with generators $r_{1}$ and $r_{2}$, and with relations

$$
r_{1}^{2}=r_{2}^{2}=\mathrm{id}, \quad r_{1} \circ r_{2}=r_{2} \circ r_{1},
$$

is a group isomorphic to $\mathbb{Z}_{2} \times \mathbb{Z}_{2}$. Note that $r_{3}=r_{1} \circ r_{2}$.

Let $s_{1}, s_{2}, s_{3}: \mathbb{K} P^{2} \rightarrow \mathbb{K} P^{2}$ be maps defined by formulas $s_{1}(x: y: z)=(x: z: y)$, $s_{2}(x: y: z)=(z: y: x)$, and $s_{3}(x: y: z)=(y: x: z)$. The set of maps $S=\left\{\right.$ id, $s_{1} \circ s_{2}$, $\left.s_{2} \circ s_{1}, s_{1}, s_{2}, s_{1} \circ s_{2} \circ s_{1}\right\}$ with the operation of composition of the maps, with generators $s_{1}$ and $s_{2}$, and with relations

$$
s_{1}^{2}=s_{2}^{2}=\mathrm{id}, \quad s_{1} \circ s_{2} \circ s_{1}=s_{2} \circ s_{1} \circ s_{2},
$$

is a group isomorphic to the symmetric group $S_{3}$. Note that $s_{3}=s_{1} \circ s_{2} \circ s_{1}$.

Let $h y$ be the birational transformation defined by the formula $h y(x: y: z)=$ $\left(x^{2}: y z: x z\right)$, whose inverse transformation is $h y^{-1}(x: y: z)=\left(x z: x y: z^{2}\right)$. Due to Newton, the transformation $h y$ is called a hyperbolism. The set $H=\left\{\ldots, h y^{-2}, h y^{-1}, \mathrm{id}\right.$, $\left.h y, h y^{2}, \ldots\right\}$ of integral powers of $h y$ is a free group isomorphic to $\mathbb{Z}$.

Let $G=R * S * H$ be the free product of groups $R, S$, and $H$. This means that the set of generators of $G$ is the union of the generators of $R, S$, and $H$, and the set of relations of $G$ is the union of the relations of $R, S$, and $H$.

DEFINITION 3.1. The factor group $G / \mathscr{R}$ with generators $r_{1}, r_{2}, s_{1}, s_{2}, h y$, where $\mathscr{R}$ is the system of relations

$$
\mathscr{R}:\left\{\begin{array}{l}
r_{1} \circ s_{1}=s_{1} \circ r_{1}, \\
r_{2} \circ s_{1}=s_{1} \circ r_{2} \circ r_{1}, \\
r_{1} \circ s_{2}=s_{2} \circ r_{1} \circ r_{2}, \\
r_{2} \circ s_{2}=s_{2} \circ r_{2}, \\
r_{1} \circ h y=h y \circ r_{1} \circ r_{2}, \\
r_{2} \circ h y=h y \circ r_{2}, \\
s_{1} \circ h y=h y \circ s_{1} \circ s_{2} \circ h y \circ s_{1}, \\
h y \circ s_{2} \circ h y=s_{2},
\end{array}\right.
$$


is called the group of birational monomial transformations of $\mathbb{K} P^{2}$ and denoted by $T\left(\mathbb{K} P^{2}\right)$.

The group of birational monomial transformations $T\left(\mathbb{K} P^{2}\right)$ is a subgroup of the Cremona group $\operatorname{Cr}\left(\mathbb{K} P^{2}\right)$ (see $\left.[2,3]\right)$.

Below in this paper, the word "transformation" without an adjective always means a "birational monomial transformation."

Every transformation $\varphi \in T\left(\mathbb{K} P^{2}\right)$ can be represented as a composition $\varphi_{1} \circ \cdots \circ \varphi_{s}$, where each of $\varphi_{1}, \ldots, \varphi_{s}$ is a positive integral power of one of the generators of the group $T\left(\mathbb{K} P^{2}\right)$, because $r_{1}^{-1}=r_{1}, r_{2}^{-1}=r_{2}, s_{1}^{-1}=s_{1}, s_{2}^{-1}=s_{2}$, and $h y^{-n}=s_{2} \circ h y^{n} \circ s_{2}$. Every transformation $\varphi$ can be represented in the form

$$
\varphi(x: y: z)=\left(\varepsilon_{1} x^{\alpha_{1}} y^{\beta_{1}} z^{\gamma_{1}}: \varepsilon_{2} x^{\alpha_{2}} y^{\beta_{2}} z^{\gamma_{2}}: x^{\alpha_{3}} y^{\beta_{3}} z^{\gamma_{3}}\right),
$$

where $\varepsilon_{1}, \varepsilon_{2} \in\{-1,1\} ; \alpha_{i}, \beta_{i}$, and $\gamma_{i}$ are nonnegative integers; and the monomials $x^{\alpha_{1}} y^{\beta_{1}} z^{\gamma_{1}}, x^{\alpha_{2}} y^{\beta_{2}} z^{\gamma_{2}}, x^{\alpha_{3}} y^{\beta_{3}} z^{\gamma_{3}}$ have no common factors. We stress this convention, for example, $(h y \circ h y)(x: y: z)=\left(x^{4}: x y z^{2}: x^{3} z\right)=\left(x^{3}: y z^{2}: x^{2} z\right)$, and accept only the last form. It means that one or two of $\alpha_{1}, \alpha_{2}, \alpha_{3}$, one or two of $\beta_{1}, \beta_{2}, \beta_{3}$, one or two of $\gamma_{1}, \gamma_{2}, \gamma_{3}$ are equal to 0 , and $\alpha_{1}+\beta_{1}+\gamma_{1}=\alpha_{2}+\beta_{2}+\gamma_{2}=\alpha_{3}+\beta_{3}+\gamma_{3}$. The integer $\alpha_{1}+\beta_{1}+\gamma_{1}$ is a degree of the transformation $\varphi$. For example, the degree of the transformations $r_{1}, r_{2}, s_{1}, s_{2}$ equals 1 , and the degree of $h y^{n}$ equals $|n|+1$, where $n \in \mathbb{Z}$.

Denote the element $s_{3} \circ h y \circ s_{3} \in T\left(\mathbb{K} P^{2}\right)$ as $h x$. Its inverse is $h x^{-1}=s_{2} \circ s_{1} \circ h y \circ$ $s_{1} \circ s_{2}$. In homogeneous coordinates it is defined by formulae $h x(x: y: z)=\left(x z: y^{2}\right.$ : $y z)$ and $h x^{-1}(x: y: z)=\left(x y: y z: z^{2}\right)$.

In the following theorem and below, the phrase "a polynomial $f(x, y, 1)$ subjected to the transformation $\varphi$ is carried onto the polynomial $l(x, y, 1)$ " means that $l(x, y, 1)=$ $\left[\left.\left(f \circ \varphi^{-1}\right)(x, y, z)\right|_{z=1}\right]^{\wedge}$.

THEOREM 3.2. Let $p$ and $q$ be mutually prime natural integers, $0<q<p$. Let $r$ and $s$ be integers which satisfy the following conditions: (1) $0<r<p, 0 \leq s<q$, (2) $r / p+s / q<1$, and (3) $r \equiv-q^{\phi(p)-1}(\bmod p)$ and $s \equiv-p^{\phi(q)-1}(\bmod q)$, where $\phi(m)$ is the Euler function. Then every polynomial $f(x, y, 1)=A x^{p}+B y^{q}+C x^{r} y^{s}$, where at least two of $A, B, C$ are not zero, subjected to the transformation

$$
\varphi=\left(h x^{\left(1-(-1)^{k+1}\right) / 2} \circ h y^{\left(1-(-1)^{k}\right) / 2}\right)^{a_{k}} \circ \ldots \circ h x^{a_{4}} \circ h y^{a_{3}} \circ h x^{a_{2}} \circ h y^{a_{1}}
$$

is carried onto the polynomial $l(x, y, 1)=A x+B y+C$, where the integers $a_{1}, a_{2}, \ldots, a_{k}$ are provided by expansion of $p / q$ in the continued fraction with adjusted last denominator

$$
\begin{aligned}
\frac{p}{q}=a_{1}+\frac{1}{a_{2}+} & \begin{array}{l}
\ddots \\
+\frac{1}{a_{k-1}+\frac{1}{\left(a_{k}+1\right)}}
\end{array}
\end{aligned}
$$

in other words, $l=\left(f \circ \varphi^{-1}\right)^{\text {. }}$. 
COROLLARY 3.3. (1) If the polynomial $f(x, y, 1)$ subjected to a transformation $\psi$ is carried onto the polynomial $\varepsilon_{1} A x+\varepsilon_{2} B y+\varepsilon_{3} C$, where $\varepsilon_{1}, \varepsilon_{2}, \varepsilon_{3} \in\{1,-1\}$, then $\psi=$ $r_{1}^{(1 / 2)\left(1-\varepsilon_{1}\right)} \circ r_{2}^{(1 / 2)\left(1-\varepsilon_{2}\right)} \circ\left(r_{1} \circ r_{2}\right)^{(1 / 2)\left(1-\varepsilon_{3}\right)} \circ \varphi$.

(2) If the polynomial $f(x, y, 1)$ subjected to a transformation $\psi$ is carried onto the polynomial $A+B x+C y, A y+B+C x, A x+B+C y, A+B y+C x$, or $A y+B x+C$, then $\psi=s_{1} \circ s_{2} \circ \varphi, \psi=s_{2} \circ s_{1} \circ \varphi, \psi=s_{1} \circ \varphi, \psi=s_{2} \circ \varphi$, or $\psi=s_{1} \circ s_{2} \circ s_{1} \circ \varphi$, respectively.

(3) If condition (2) of Theorem 3.2 is changed to condition ( $\left.2^{\prime}\right), r / p+s / q>1$, and other conditions and notations are kept, and if the polynomial $f(x, y, 1)$ subjected to a transformation $\psi$ is carried onto the polynomial $A x+B y+C$, then $\psi=\varphi \circ \operatorname{tr}=\operatorname{tr} \circ \varphi$, where $\operatorname{tr}=s_{1} \circ h y^{-1} \circ s_{1} \circ s_{2} \circ s_{1} \circ h y$ is well-known standard (triangular) quadratic transformation $\operatorname{tr}(x: y: z)=(y z: x z: x y)$.

REMARK 3.4. There is only one more possible case: when $p=q=1$, which does not satisfy the theorem. In this case either $r=s=0$, and the polynomial $A x+B y+C$ is carried onto itself by the identity transformation: $\varphi=\mathrm{id}$, or $r=s=1$, and the polynomial $A x+B y+C x y$ is carried onto the polynomial $A x+B y+C$ by the transformation $\varphi=s_{3} \circ \operatorname{tr}=s_{3} \circ s_{1} \circ h y^{-1} \circ s_{1} \circ s_{2} \circ s_{1} \circ h y$.

4. Proof of the theorem. A birational monomial transformation $\psi$ maps a polynomial $f$ onto a polynomial $\left(f \circ \psi^{-1}\right)$. We find the connection between $N\left(f \circ \psi^{-1}\right)$ and $N(f)$.

Every transformation $\psi^{-1}$ written in the form (3.5) induces a generic linear mapping $A(\psi): \mathbb{R}^{2} \rightarrow \mathbb{R}^{2}$ of the plane of Newton's polygons, which can be defined on any monomial. Namely, if $g$ is a monomial, say $g(x, y, z)=x^{\omega_{1}} y^{\omega_{2}} z^{\omega_{3}}$, then

$$
\left(g \circ \psi^{-1}\right)(x, y, z)=x^{\alpha_{1} \omega_{1}+\alpha_{2} \omega_{2}+\alpha_{3} \omega_{3}} y^{\beta_{1} \omega_{1}+\beta_{2} \omega_{2}+\beta_{3} \omega_{3}} z^{\gamma_{1} \omega_{1}+\gamma_{2} \omega_{2}+\gamma_{3} \omega_{3}},
$$

thus, the linear mapping $A(\psi)$ is defined by the matrix

$$
A_{\psi}=\left(\begin{array}{lll}
\alpha_{1} & \alpha_{2} & \alpha_{3} \\
\beta_{1} & \beta_{2} & \beta_{3} \\
\gamma_{1} & \gamma_{2} & \gamma_{3}
\end{array}\right) .
$$

And thus, $N\left(f \circ \psi^{-1}\right)=A_{\psi}(N(f))$ for every polynomial $f$.

Remark that the generators of the birational monomial group have matrices

$$
\begin{gathered}
A_{\mathrm{id}}=A_{r_{1}}=A_{r_{2}}=\left(\begin{array}{lll}
1 & 0 & 0 \\
0 & 1 & 0 \\
0 & 0 & 1
\end{array}\right), \\
A_{s_{1}}=\left(\begin{array}{lll}
1 & 0 & 0 \\
0 & 0 & 1 \\
0 & 1 & 0
\end{array}\right), \quad A_{s_{2}}=\left(\begin{array}{lll}
0 & 0 & 1 \\
0 & 1 & 0 \\
1 & 0 & 0
\end{array}\right), \quad A_{h y}=\left(\begin{array}{lll}
2 & 0 & 1 \\
0 & 1 & 0 \\
0 & 1 & 1
\end{array}\right) .
\end{gathered}
$$

The set of matrices $A\left(T\left(\mathbb{K} P^{2}\right)\right)=\left\{A_{\varphi} \mid \varphi \in T\left(\mathbb{K} P^{2}\right)\right\}$ is a subset in the linear group $G L(3, \mathbb{R})$ of $3 \times 3$-matrices. The operation $\diamond$ defined by the formula $A_{\varphi} \diamond A_{\psi}=A_{\varphi \circ \psi}$ converts the set $A\left(T\left(\mathbb{K} P^{2}\right)\right)$ into a group, which is natural $\left(\varphi \mapsto A_{\varphi}\right.$ ) homomorphic image of $T\left(\mathbb{K} P^{2}\right)$. 
It is clear that every birational monomial transformation $\varphi$ induces one-to-one correspondence between monomials of the polynomials $f$ and $\left(f \circ \varphi^{-1}\right)^{\wedge}$. Thus, to represent a transformation $\varphi$ as a composition of generators of birational monomial group, it is enough to study the action of the transformation $\varphi$ on a polynomial $f$ whose Newton's polygon $N(f)$ is a triangle with the area $1 / 2$.

We consider a polynomial $f(x, y, 1)=A x^{p}+B y^{q}+C x^{r} y^{s}$, with $A B C \neq 0$, where $p$ and $q$ are mutually prime integers, $0<q<p$, and $r$ and $s$ are integers, which satisfy the following conditions: (1) $0<r<p, 0 \leq s<q$, (2) $r / p+s / q<1$, and (3) $r \equiv-q^{\phi(p)-1}(\bmod p)$ and $s \equiv-p^{\phi(q)-1}(\bmod q)$, where $\phi(m)$ is the Euler function. The Newton polygon $N(f)$ is the triangle with integer vertices $(p, 0),(0, q),(r, s)$ which has no other integer points belonging to its interior and boundary but its three vertices. According to the Pick theorem [7], the area of such a triangle equals $1 / 2$. The genus of any curve $f(x, y, 1)=A x^{p}+B y^{q}+C x^{r} y^{s}=0$ with such properties is zero and thus, all such curves are birationally equivalent.

Note that $h y^{-a}(x: y: 1)=\left(x: x^{a} y: 1\right)$ and $h x^{-a}(x: y: 1)=\left(x y^{a}: y: 1\right)$. We evaluate $\left(f \circ \varphi^{-1}\right)(x, y, 1)$ as follows.

The first step.

$$
\begin{aligned}
\left(f \circ h y^{-a_{1}}\right)(x, y, 1) & =f\left(x, x^{a_{1}} y, 1\right)=x^{a_{1} q}\left(A x^{b_{1}}+B y^{q}+C x^{c+a_{1} d-a_{1} q} y^{d}\right) \\
& =x^{u_{1}} y^{v_{1}}\left(A x^{b_{1}}+B y^{q}+C x^{c_{1}} y^{d_{1}}\right),
\end{aligned}
$$

where $u_{1}=a_{1} q, v_{1}=0, c_{1}=c+a_{1} d-a_{1} q$, and $d_{1}=d$.

The second step.

$$
\begin{aligned}
\left(f \circ h y^{-a_{1}} \circ h x^{-a_{2}}\right)(x, y, 1) & =\left(f \circ h y^{-a_{1}}\right)\left(x y^{a_{2}}, y, 1\right) \\
& =x^{u_{1}} y^{a_{2} u_{1}+v_{1}+a_{2} b_{1}}\left(A x^{b_{1}}+B y^{b_{2}}+C x^{c_{1}} y^{a_{2} c_{1}+d_{1}-a_{2} b_{1}}\right) \\
& =x^{u_{2}} y^{v_{2}}\left(A x^{b_{1}}+B y^{b_{2}}+C x^{c_{2}} y^{d_{2}}\right),
\end{aligned}
$$

where $u_{2}=u_{1}, v_{2}=a_{2} u_{1}+v_{1}+a_{2} b_{1}, c_{2}=c_{1}$, and $d_{2}=a_{2} c_{1}+d_{1}-a_{2} b_{1}$.

The third step.

$$
\begin{aligned}
\left(f \circ h y^{-a_{1}} \circ h x^{-a_{2}} \circ h y^{-a_{3}}\right)(x, y, 1) & =\left(f \circ h y^{-a_{1}} \circ h x^{-a_{2}}\right)\left(x, x^{a_{3}} y, 1\right) \\
& =x^{u_{3}} y^{v_{3}}\left(A x^{b_{3}}+B y^{b_{2}}+C x^{c_{3}} y^{d_{3}}\right),
\end{aligned}
$$

where $u_{3}=u_{2}+a_{2} v_{2}+a_{3} b_{2}, v_{3}=v_{2}, c_{3}=c_{2}+a_{3} d_{2}-a_{3} b_{2}$, and $d_{3}=d_{2}$.

The fourth step.

$$
\begin{aligned}
& \left(f \circ h y^{-a_{1}} \circ h x^{-a_{2}} \circ h y^{-a_{3}} \circ h x^{-a_{4}}\right)(x, y, 1) \\
& \quad=\left(f \circ h y^{-a_{1}} \circ h x^{-a_{2}} \circ h y^{-a_{3}}\right)\left(x y^{a_{4}}, y, 1\right) \\
& \quad=x^{u_{1}} y^{a_{2} u_{1}+v_{1}+a_{2} b_{1}}\left(A x^{b_{1}}+B y^{b_{2}}+C x^{c_{1}} y^{a_{2} c_{1}+d_{1}-a_{2} b_{1}}\right) \\
& \quad=x^{u_{2}} y^{v_{2}}\left(A x^{b_{1}}+B y^{b_{2}}+C x^{c_{2}} y^{d_{2}}\right),
\end{aligned}
$$

where $u_{2}=u_{1}, v_{2}=a_{2} u_{1}+v_{1}+a_{2} b_{1}, c_{2}=c_{1}$, and $d_{2}=a_{2} c_{1}+d_{1}-a_{2} b_{1}$. We then proceed until the $(k-1)$ th step. 
The $(k-1)$ th step. We have two cases.

The first case: $k$ is even.

$$
\begin{aligned}
& \left(f \circ h y^{-a_{1}} \circ h x^{-a_{2}} \circ \ldots \circ h x^{\left.-a_{k-2} \circ h y^{-a_{k-1}}\right)(x, y, 1)}\right. \\
& =\left(f \circ h y^{-a_{1}} \circ h x^{-a_{2}} \circ \cdots \circ h x^{-a_{k-2}}\right)\left(x, x^{a_{k-1}} y, 1\right) \\
& =x^{u_{k-1}} y^{v_{k-1}}\left(A x+B y^{b_{k-2}}+C x^{c_{k-1}} y^{d_{k-1}}\right) \text {, }
\end{aligned}
$$

where $u_{k-1}=u_{k-2}+a_{k-1} v_{k-2}+a_{k-1} b_{k-2}, v_{k-1}=v_{k-2}, c_{k-1}=c_{k-2}+a_{k-1} d_{k-2}-$ $a_{k-1} b_{k-2}$, and $d_{k-1}=d_{k-2}$.

The second case: $k$ is odd.

$$
\begin{aligned}
& \left(f \circ h y^{-a_{1}} \circ h x^{-a_{2}} \circ \cdots \circ h y^{-a_{k-2}} \circ h x^{-a_{k-1}}\right)(x, y, 1) \\
& =\left(f \circ h y^{-a_{1}} \circ h x^{-a_{2}} \circ \cdots \circ h y^{-a_{k-2}}\right)\left(x y^{a_{k-1}}, y, 1\right) \\
& =x^{u_{k-1}} y^{v_{k-1}}\left(A x^{b_{k-2}}+B y+C x^{c_{k-1}} y^{d_{k-1}}\right) \text {, }
\end{aligned}
$$

where $u_{k-1}=u_{k-2}, v_{k-1}=a_{k-1} u_{k-2}+v_{k-2}+a_{k-1} b_{k-2}, c_{k-1}=c_{k-2}$, and $d_{k-1}=a_{k-1} c_{k-2}+$ $d_{k-2}-a_{k-1} b_{k-2}$.

The kth step. We have two cases.

The first case: $k$ is even.

$$
\begin{aligned}
& \left(f \circ h y^{-a_{1}} \circ h x^{-a_{2}} \circ \cdots \circ h y^{-a_{k-1}} \circ h x^{-a_{k}}\right)(x, y, 1) \\
& \quad=\left(f \circ h y^{-a_{1}} \circ h x^{-a_{2}} \cdots \cdots h y^{-a_{k-1}}\right)\left(x y^{a_{k}}, y, 1\right) \\
& \quad=x^{u_{k}} y^{v_{k}}\left(A x+B y+C x^{c_{k}} y^{d_{k}}\right),
\end{aligned}
$$

where $u_{k}=u_{k-1}, v_{k}=a_{k} u_{k-1}+v_{k-1}+a_{k}, c_{k}=c_{k-1}$, and $d_{k}=a_{k} c_{k-1}+d_{k-1}-a_{k}$.

The second case: $k$ is odd.

$$
\begin{aligned}
& \left(f \circ h y^{-a_{1}} \circ h x^{-a_{2}} \circ \cdots \circ h x^{-a_{k-1}} \circ h y^{-a_{k}}\right)(x, y, 1) \\
& \quad=\left(f \circ h y^{-a_{1}} \circ h x^{-a_{2}} \cdots \cdots h x^{-a_{k-2}}\right)\left(x, x^{a_{k}} y, 1\right) \\
& \quad=x^{u_{k}} y^{v_{k}}\left(A x+B y+C x^{c_{k}} y^{d_{k}}\right),
\end{aligned}
$$

where $u_{k}=u_{k-1}+a_{k} v_{k-1}+a_{k}, v_{k}=v_{k-1}, c_{k}=c_{k-1}+a_{k} d_{k-1}-a_{k}$, and $d_{k}=d_{k-1}$.

This calculation shows that the integers $a_{1}, a_{2}, a_{3}, \ldots, a_{k}$ satisfy the Euclidean algorithm with adjusted last row

$$
\begin{aligned}
p & =a_{1} q+b_{1}, \\
q & =a_{2} b_{1}+b_{2}, \\
b_{1} & =a_{3} b_{2}+b_{3}, \\
\vdots & \\
b_{k-4} & =a_{k-2} b_{k-3}+b_{k-2}, \\
b_{k-3} & =a_{k-1} b_{k-2}+1, \\
b_{k-2} & =a_{k}+1,
\end{aligned}
$$

which provides the desired continued fraction. 
ACKNOWLEDGMENT. I thank Inna Korchagina (Rutgers University), David Weinberg (Texas Tech University), and Victor Zvonilov (Syktyvkar State University, Russia) for several helpful discussions.

\section{REFERENCES}

[1] B. Chevallier, Singularités et topologies optimales des hypersurfaces algébriques réelles de petites dimensions, These d'État, Université Paris VII, Paris, 1996.

[2] L. Godeaux, Les Transformations Birationnelles du Plan, Gauthier-Villars, Paris, 1927.

[3] H. P. Hudson, Cremona Transformation in Plane and Space, Cambridge University Press, Massachusetts, 1927.

[4] V. M. Kharlamov, Topological types of nonsingular surfaces of degree 4 in $\mathbf{R P}^{3}$, Funkcional. Anal. i Priložen. 10 (1976), no. 4, 55-68 (Russian).

[5] __ Isotopic types of nonsingular surfaces of degree 4 in $\mathrm{RP}^{3}$, Funkcional. Anal. i Priložen. 12 (1978), no. 1, 86-87 (Russian).

[6] A. B. Korchagin, Smoothing of 6-fold singular points and constructions of 9th degree Mcurves, Topology of Real Algebraic Varieties and Related Topics, Amer. Math. Soc. Transl. Ser. 2, vol. 173, American Mathematical Society, Rhode Island, 1996, pp. 141155.

[7] Yu. A. Shashkin, The Euler Characteristic, Popular Lectures on Mathematics, vol. 58, Nauka, Moscow, 1984.

[8] O. Ya. Viro, Gluing algebraic hypersurfaces, removing singularities, and construction of curves, Proc. International Topological Conference (Leningrad, 1982), Nauka, Moscow, 1983, pp. 149-197.

[9] __ Gluing of plane real algebraic curves and constructions of curves of degrees 6 and 7 , Topology (Leningrad, 1982), Lecture Notes in Math., vol. 1060, Springer, Berlin, 1984, pp. 187-200.

Anatoly B. Korchagin: Department of Mathematics and Statistics, Texas Tech University, Lubbock, TX 79409-1042, USA

E-mail address: korchag@math.ttu.edu 


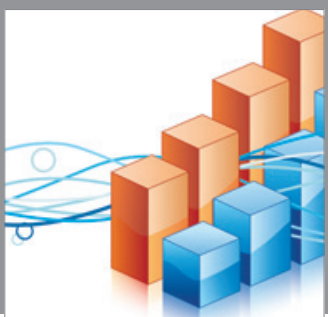

Advances in

Operations Research

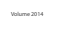

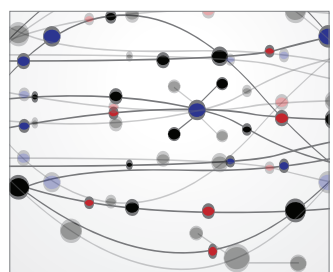

\section{The Scientific} World Journal
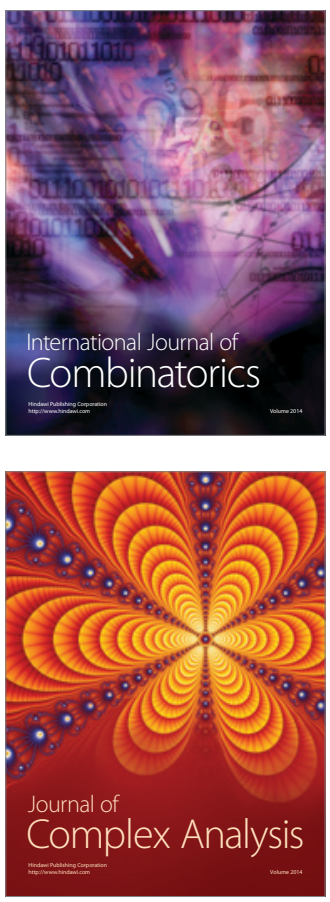

International Journal of

Mathematics and

Mathematical

Sciences
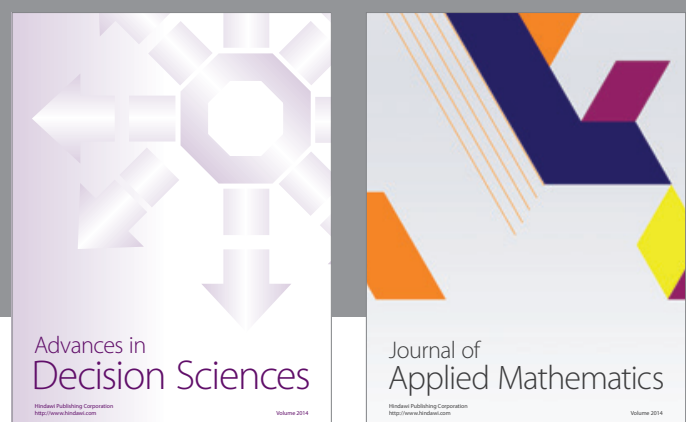

Journal of

Applied Mathematics
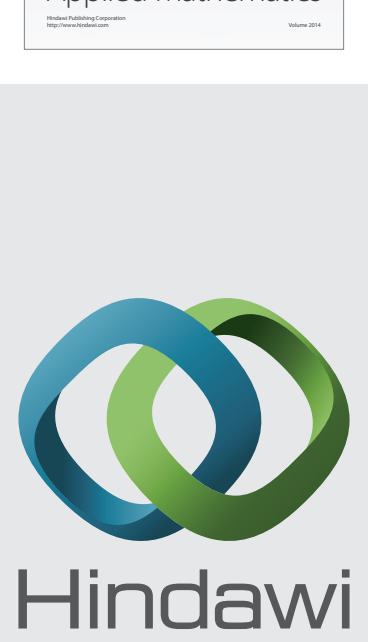

Submit your manuscripts at http://www.hindawi.com
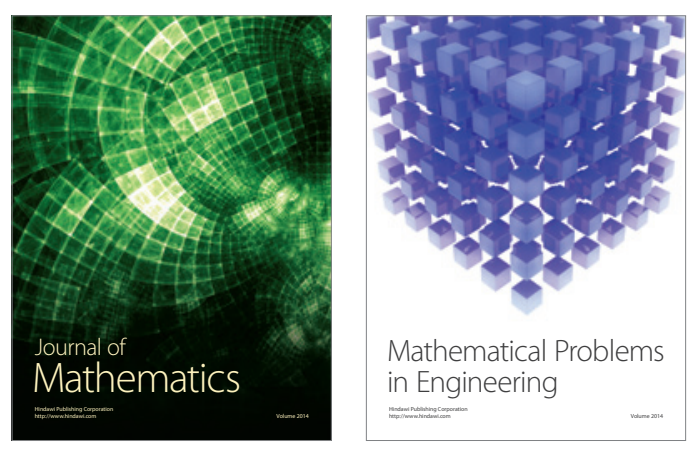

Mathematical Problems in Engineering
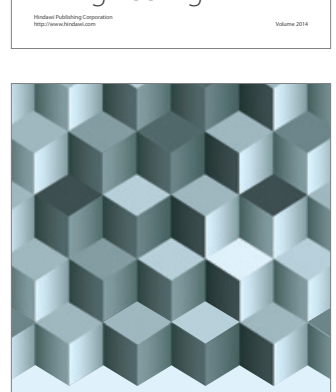

Journal of

Function Spaces
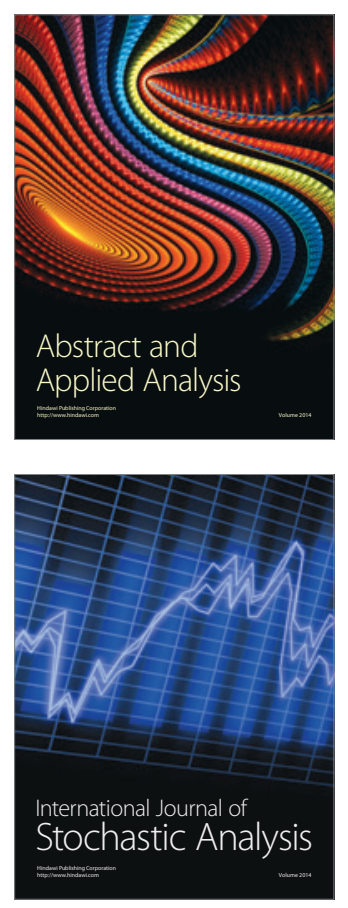

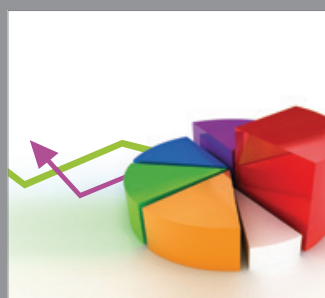

ournal of

Probability and Statistics

Promensencen
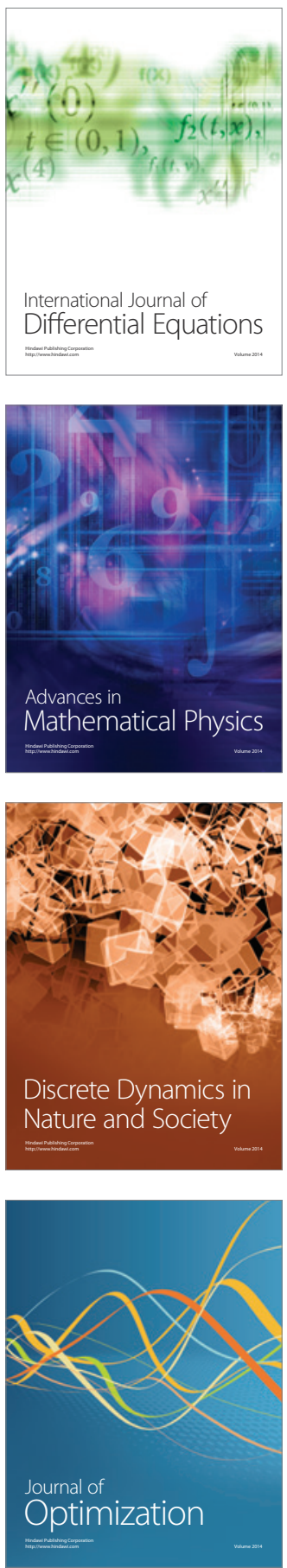